\title{
Reductions in brain pericytes are associated with arteriovenous malformation vascular instability
}

\author{
Ethan A. Winkler, MD, PhD, ${ }^{1,2}$ Harjus Birk, MD, ${ }^{1}$ Jan-Karl Burkhardt, MD, ${ }^{1}$ Xiaolin Chen, MD, 2,3 \\ John K. Yue, BS, ${ }^{1}$ Diana Guo, BA, ${ }^{2}$ W. Caleb Rutledge, MD, ${ }^{1,2}$ George F. Lasker, MD, PhD, ${ }^{1}$ \\ Carlene Partow, BS, ${ }^{1}$ Tarik Tihan, MD, PhD, ${ }^{4}$ Edward F. Chang, MD, ${ }^{1}$ Hua Su, MD, ${ }^{2}$ Helen Kim, PhD, ${ }^{2}$ \\ Brian P. Walcott, MD, 1,2,5 and Michael T. Lawton, MD ${ }^{1,2}$
}

\begin{abstract}
${ }^{1}$ Department of Neurological Surgery; ${ }^{2}$ Center for Cerebrovascular Research, Department of Anesthesia and Perioperative Care; and ${ }^{4}$ Department of Pathology, University of California, San Francisco; ${ }^{5}$ Department of Neurological Surgery, University of Southern California, Los Angeles, California; and 'Department of Neurological Surgery, Beijing Tiantan Hospital, Capital Medical University, Beijing, People's Republic of China
\end{abstract}

\begin{abstract}
OBJECTIVE Brain arteriovenous malformations (bAVMs) are rupture-prone tangles of blood vessels with direct shunting of blood flow between arterial and venous circulations. The molecular and/or cellular mechanisms contributing to bAVM pathogenesis and/or destabilization in sporadic lesions have remained elusive. Initial insights into AVM formation have been gained through models of genetic AVM syndromes. And while many studies have focused on endothelial cells, the contributions of other vascular cell types have yet to be systematically studied. Pericytes are multifunctional mural cells that regulate brain angiogenesis, blood-brain barrier integrity, and vascular stability. Here, the authors analyze the abundance of brain pericytes and their association with vascular changes in sporadic human AVMs.

METHODS Tissues from bAVMs and from temporal lobe specimens from patients with medically intractable epilepsy (nonvascular lesion controls [NVLCs]) were resected. Immunofluorescent staining with confocal microscopy was performed to quantify pericytes (platelet-derived growth factor receptor-beta [PDGFRß] and aminopeptidase N [CD13]) and extravascular hemoglobin. Iron-positive hemosiderin deposits were quantified with Prussian blue staining. Syngo iFlow post-image processing was used to measure nidal blood flow on preintervention angiograms.
\end{abstract}

RESULTS Quantitative immunofluorescent analysis demonstrated a $68 \%$ reduction in the vascular pericyte number in bAVMs compared with the number in NVLCs $(p<0.01)$. Additional analysis demonstrated $52 \%$ and $50 \%$ reductions in the vascular surface area covered by CD13- and PDGFRß-positive pericyte cell processes, respectively, in bAVMs ( $p$ $<0.01)$. Reductions in pericyte coverage were statistically significantly greater in bAVMs with prior rupture $(p<0.05)$. Unruptured bAVMs had increased microhemorrhage, as evidenced by a 15.5-fold increase in extravascular hemoglobin compared with levels in NVLCs ( $<$ 0.01). Within unruptured bAVM specimens, extravascular hemoglobin correlated negatively with pericyte coverage (CD13: $r=-0.93, p<0.01$; PDGFR $: r=-0.87, p<0.01)$. A similar negative correlation was observed with pericyte coverage and Prussian blue-positive hemosiderin deposits $(C D 13: r=-0.90, p<0.01$; PDGFR $: r=-0.86, p<0.01)$. Pericyte coverage positively correlated with the mean transit time of blood flow or the time that circulating blood spends within the bAVM nidus (CD13: $r=0.60, p<0.05$; PDGFR : $r=0.63, p<0.05$ ). A greater reduction in pericyte coverage is therefore associated with a reduced mean transit time or faster rate of blood flow through the bAVM nidus. No correlations were observed with time to peak flow within feeding arteries or draining veins.

CONCLUSIONS Brain pericyte number and coverage are reduced in sporadic bAVMs and are lowest in cases with prior rupture. In unruptured bAVMs, pericyte reductions correlate with the severity of microhemorrhage. A loss of pericytes also correlates with a faster rate of blood flow through the bAVM nidus. This suggests that pericytes are associated with and may contribute to vascular fragility and hemodynamic changes in bAVMs. Future studies in animal models are needed to better characterize the role of pericytes in AVM pathogenesis.

https://thejns.org/doi/abs/10.3171/2017.6.JNS17860

KEY WORDS pericytes; arteriovenous malformations; blood-brain barrier; microhemorrhage; intracerebral hemorrhage; stroke; vascular disorders

ABBREVIATIONS bAVM = brain arteriovenous malformation; $\mathrm{BBB}=$ blood-brain barrier; $\mathrm{CD} 13$ = aminopeptidase $\mathrm{N}$; CD31 = platelet endothelial adhesion molecule 1; GFAP = glial fibrillary acidic protein; MTT = mean transit time; NVLC = nonvascular lesion control; PDGFR = platelet-derived growth factor receptor-beta; ROI = region of interest.

SUBMITTED April 5, 2017. ACCEPTED June 19, 2017.

INCLUDE WHEN CITING Published online January 5, 2018; DOI: 10.3171/2017.6.JNS17860. 
$\mathrm{B}$ RAIN arteriovenous malformations (bAVMs) are rupture-prone tangles of blood vessels with direct shunting of blood flow between arterial and venous circulations without a clear intervening capillary bed. ${ }^{18,26,43}$ Rates of intracerebral hemorrhage range from $1 \%$ to $3 \%$ per person-year, often with catastrophic neurological consequences. ${ }^{11}$ Numerous structural abnormalities suggestive of instability have been described, including dilated perinidal capillary networks, $, 428,34$ intranidal or feeding artery aneuryms,${ }^{11}$ and/or microhemorrhage. ${ }^{1,12,24}$ However, the cellular and/or molecular mechanisms underlying bAVM destabilization or rupture have remained elusive.

Normal cerebrovascular structure and function depend on the coordinated signaling of multiple interconnected cell types, including endothelial cells, mural cells (vascular smooth muscle cells and pericytes), immune cells, glia, and neurons. ${ }^{8,34}$ Pericytes are the principal mural cell population of the cerebral microvasculature, covering roughly $80 \%-90 \%$ of the vascular wall. ${ }^{6,38,40}$ Pericytes fulfill a modulatory role in a number of integral cerebrovascular functionalities, which are disrupted in bAVMs, including regulation of brain angiogenesis, endothelial proliferation, vascular diameter, blood flow regulation, vascular wall stability, and integrity of the blood-brain barrier (BBB). ${ }^{2,31,44}$ Qualitative studies have suggested that pericytes may be reduced in human bAVMs and/or rodent models of bAVM ${ }^{8,34}$ However, pericytes have yet to be systematically studied, and many published reports have focused exclusively on the endothelium.

In the present study, we quantified pericyte abundance in resected sporadic human bAVMs and studied their association with vascular changes. We report not only that pericytes are reduced in bAVMs, but also that the reductions are associated with indices of instability, for example, clinically significant rupture and microhemorrhage, and alterations in nidal blood flow.

\section{Methods \\ Human Subjects}

Tissue specimens were obtained from bAVMs that had been resected by the senior author (M.T.L.). For nonvascular lesion controls (NVLCs), temporal lobe specimens were similarly acquired from subjects undergoing anterior temporal lobectomy for medically refractory epilepsy (E.F.C.). All tissues were immersion fixed in formaldehyde and embedded in paraffin blocks for neuropathological analysis. In total, $30 \mathrm{bAVM}$ tissue specimens were obtained from neuropathology, but 10 samples were either too small or of too poor quality to be included in our analysis. There were 11 control samples. A summary of subject characteristics is provided in Table 1 . This study was approved by the University of California, San Francisco, Institutional Review Board and performed in compliance with the Health Insurance Portability and Accountability Act regulations.

\section{Immunofluorescent Analysis}

Brain pericyte and hemoglobin immunodetection was

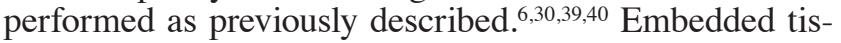
sue specimens were sectioned using a microtome at a thickness of $6 \mu \mathrm{m}$. Sections were then deparaffinized and rehydrated with serial ethanol washes to distilled water. All sections were boiled in target antigen retrieval solution, $\mathrm{pH} 9$ (Dako), for 20 minutes in a conventional microwave. Following antigen retrieval, tissue sections were blocked in $10 \%$ normal swine serum (Vector Laboratories) with $0.05 \%$ Triton X-100 (Sigma-Aldrich) for 1 hour at room temperature. Sections were then incubated with the following primary antibodies at $4^{\circ} \mathrm{C}$ : goat anti-human platelet-derived growth factor receptor-beta (PDGFR $\beta$; 1:100, R\&D Systems), mouse anti-human aminopeptidase N (CD13; 1:100, R\&D Systems), goat antihemoglobin (10 $\mu \mathrm{g} / \mathrm{ml}, \mathrm{R} \& \mathrm{D}$ Systems), mouse anti-human glial fibrillary acidic protein (GFAP; Cell Signaling Inc.), and/or rabbit anti-human platelet endothelial adhesion molecule 1 (CD31; 1:25, Abcam Inc.). Sections were then washed in phosphate-buffered saline containing $0.05 \%$ Triton X-100 and incubated in secondary antibody for 1 hour at room temperature. To visualize pericytes, sections were incubated in bovine anti-goat Cy3 (Jackson ImmunoResearch Laboratories Inc.) and donkey anti-mouse Alexa Fluor 488 (Jackson Laboratories) antibodies to detect PDGFR $\beta$ and CD13, respectively. To visualize astrocytes, sections were incubated with donkey anti-mouse Alexa Fluor 488 (Jackson Laboratories) antibodies to detect GFAP. To visualize extravascular hemoglobin, sections were incubated in bovine anti-goat Cy3 antibody (Jackson Laboratories). To visualize endothelium, sections were incubated in biotinylated horse anti-rabbit antibody (Jackson Laboratories) or biotinylated Ulex europaeus agglutinin 1 (lectin, Vector Laboratories), followed by incubation in DyLight 649-conjugated streptavidin (Vector Laboratories). All sections were incubated in $1 \%$ Sudan Black B diluted in $70 \%$ ethanol for 10 minutes at room temperature to quench autofluorescence.

Following completion of immunodetection, tissue sections were mounted with fluorescent mounting medium (Dako) and coverslipped. All imaging was performed with a Leica TCS SP5 X confocal microscope utilizing a 20x objective (Leica Microsystems Inc.). For all imaging, identical laser settings were used for AVMs and NVLCs. Representative images were prepared with NIH ImageJ software.

\section{Image Analysis}

All analyses were performed with NIH ImageJ software. Ten- to $12-\mu \mathrm{m}$ maximum projection z-stack images were reconstructed. For all studies, 3-5 randomly selected fields in 3 nonadjacent tissue sections per tissue specimen were analyzed as previously described. ${ }^{30,39}$ For pericyte coverage analysis, PDGFR $\beta$ - or CD13-positive surface area was measured with the ImageJ Area measurement tool and divided by the CD31-positive endothelial surface area as previously described. ${ }^{6,30,38,40}$ For pericyte number analysis, pericyte cell bodies were manually counted and divided by the CD31-positive endothelial surface area as previously described. ${ }^{6,30,38,40}$ Extravascular hemoglobin was quantified as previously described. ${ }^{39,40}$ The intravascular hemoglobin-positive immunofluorescent signal was subtracted from maximum projection z-stack images utilizing the ImageJ colocalization function as previously de- 
TABLE 1. Clinical characteristics of subjects providing tissue samples

\begin{tabular}{|c|c|c|c|c|c|}
\hline \multirow[b]{2}{*}{ Parameter } & \multirow[b]{2}{*}{ NVLCs } & \multicolumn{3}{|c|}{ AVMs } & \multirow[b]{2}{*}{ p Value* } \\
\hline & & Total & Unruptured & Ruptured & \\
\hline No. of specimens & 11 & 20 & 12 & 8 & \\
\hline Mean age in yrs & $32.2 \pm 7.0$ & $33.2 \pm 18.4$ & $36.2 \pm 16.0$ & $28.6 \pm 21.9$ & 0.38 \\
\hline Sex & $3 \mathrm{M} / 8 \mathrm{~F}$ & $10 \mathrm{M} / 10 \mathrm{~F}$ & $7 \mathrm{M} / 5 \mathrm{~F}$ & $3 \mathrm{M} / 5 \mathrm{~F}$ & 0.65 \\
\hline Lesion location & & & & & 0.26 \\
\hline Supratentorial & $11(100)$ & $16(80)$ & $11(92)$ & $5(62.5)$ & \\
\hline Infratentorial & $0(0)$ & $4(20)$ & $1(8)$ & $3(37.5)$ & \\
\hline \multicolumn{6}{|l|}{ Lesion characteristics } \\
\hline Nidus size & - & $1.9 \pm 0.8$ & $2.2 \pm 0.6$ & $1.6 \pm 1.1$ & 0.12 \\
\hline Deep venous drainage & - & $6(30)$ & $4(33)$ & $2(25)$ & 1.0 \\
\hline Eloquence & - & $10(50)$ & $6(50)$ & $4(50)$ & 1.0 \\
\hline \multicolumn{6}{|l|}{ Spetzler-Martin grade } \\
\hline Mean & - & $2 \pm 0.7$ & $1.9 \pm 0.7$ & $2.1 \pm 0.8$ & 0.54 \\
\hline I & - & $5(25)$ & $3(25)$ & $2(25)$ & \\
\hline II & - & $10(50)$ & $7(58)$ & $3(37.5)$ & \\
\hline III & - & $5(25)$ & $2(17)$ & $3(37.5)$ & \\
\hline IV & - & $0(0)$ & $0(0)$ & $0(0)$ & \\
\hline V & - & $0(0)$ & $0(0)$ & $0(0)$ & \\
\hline \multicolumn{6}{|l|}{ Supplemental grade $†$} \\
\hline Mean & - & $2.8 \pm 1.1$ & $3.3 \pm 1.0$ & $2.1 \pm 0.8$ & 0.01 \\
\hline 1 & - & $2(10)$ & $0(0)$ & $2(25)$ & \\
\hline 2 & - & $6(30)$ & $3(25)$ & $3(37.5)$ & \\
\hline 3 & - & $6(30)$ & $3(25)$ & $3(37.5)$ & \\
\hline 4 & - & $5(25)$ & $5(42)$ & $0(0)$ & \\
\hline 5 & - & $1(5)$ & $1(8)$ & $0(0)$ & \\
\hline Presenting symptom & & & & & 0.01 \\
\hline Seizure & $11(100)$ & $6(30)$ & $6(50)$ & $0(0)$ & \\
\hline Headache & - & $9(45)$ & $5(42)$ & $4(50)$ & \\
\hline Altered mental status & - & $4(20)$ & $0(0)$ & $4(50)$ & \\
\hline Incidental lesion & - & $1(5)$ & $1(8)$ & $0(0)$ & \\
\hline \multicolumn{6}{|l|}{ Presurgical treatment } \\
\hline Embolization & - & $7(35)$ & $3(15)$ & $4(50)$ & 0.36 \\
\hline Radiosurgery & - & $1(5)$ & $0(0)$ & $1(8)$ & 0.40 \\
\hline $\begin{array}{l}-=\text { not applicable. } \\
\text { Values are expressed as the mea } \\
\text { * Comparison between unrupture } \\
\dagger \text { For additional information on th } \\
\text { tary grading scale for selecting }\end{array}$ & $\begin{array}{l}\text { רdard error of } \\
\text { uptured AVMs } \\
\text { ementary gra } \\
\text { vith brain arte }\end{array}$ & $\begin{array}{l}\text { an or as numbe } \\
\text { ale, see Lawton } \\
\text { us malformatior }\end{array}$ & $\mathrm{H}$, McCulloch & lak B, Young V & upplemen- \\
\hline
\end{tabular}

scribed..$^{40}$ The remaining extravascular hemoglobin-positive immunofluorescent signal was subjected to threshold processing and quantified with ImageJ Integrated Density analysis. Variability in background staining was minimized with post-image thresholding. All image analyses were performed by an investigator blinded to the pathological diagnosis to avoid the introduction of bias.

\section{Hemosiderin Deposits}

Formaldehyde-fixed paraffin-embedded tissue sections were deparaffinized and rehydrated. Prussian blue staining was then performed as described by the manufacturer utilizing an iron staining kit (Abcam Inc.). Nuclear fast red was used as a counterstain. Sections were subsequently coverslipped using Shandon-Mount mounting medium (Thermo Scientific) and imaged with a Leica DMI6000 inverted epifluorescence microscope (Leica Microsystems Inc.). Prussian blue hemosiderin deposits were manually counted by a blinded investigator and expressed per square millimeter of tissue.

\section{AVM Blood Flow Analysis}

Syngo iFlow post-image processing software (Siemens) was used to reconstruct color-coded preoperative digital subtraction angiograms (Axiom Artis, Siemens) when available. The syngo iFlow software condenses the 
complete digital subtraction angiography run into a single color-coded image to facilitate quantification of hemodynamic parameters. A region of interest (ROI) was manually drawn over the caliber of selected vessels. A time versus intensity graph was generated, which permitted quantification of the ROI peak time (Tmax) - this is defined as the time to peak contrast intensity in the selected vascular ROI. Nidal mean transit time (MTT) was next figured by calculating the difference between the Tmax of the closest arterial feeding artery and venous outflow in the draining vein. In the instance of multiple feeding arteries and/or draining veins, the fastest MTT of the nidus was selected for each subject. The MTT is a measure of the time that circulating blood spends within the bAVM nidus; therefore, a higher MTT suggests a slower rate of flow, whereas a lower MTT suggests a faster rate of blood flow.

\section{Statistical Analysis}

All continuous variables were analyzed with the Student t-test to determine differences between AVMs and NVLCs. For categorical variables, either a Fisher's exact test or chi-square test was performed to analyze differences between groups. Pearson's correlation coefficient (r) was used for all correlations. A p value $<0.05$ was considered the threshold for statistical significance in all analyses. Values are presented as the mean \pm standard error of the mean unless otherwise specified.

\section{Results}

\section{Pericytes Reduced in Human bAVMs}

Pericytes are perivascular cells embedded in a common vascular basement membrane on the abluminal endothelial cell membrane and comprise both a cell body and stellate-shaped finger-like cell processes, which en- sheathe the endothelial vascular tube (Fig. 1). Using established methodologies, we quantified both pericyte number and coverage. In total, reductions in vascular pericytes as measured by both parameters were observed in all 20 bAVM specimens. Quantitative analysis demonstrated a statistically significant $68 \%$ reduction in the CD13-positive pericyte number in bAVMs compared with the number in NVLCs (bAVMs $862.2 \pm 47.3$ pericytes per $\mathrm{mm}^{2}$ vascular surface area; NVLCs $2657.0 \pm 140.7$ pericytes per $\mathrm{mm}^{2}$ vascular surface area; $\mathrm{p}<0.01$; Fig. $2 \mathrm{~A}$ and $\mathrm{B}$ ). Similar analysis utilizing the independent pericyte cell marker PDGFR $\beta$ confirmed a statistically significant $68 \%$ reduction in the pericyte cell number between bAVMs and NVLCs (bAVMs $845.6 \pm 47.9$ pericytes per $\mathrm{mm}^{2}$ vascular surface area; NVLCs $2658.1 \pm 174.8$ pericytes per $\mathrm{mm}^{2}$ vascular surface area; $\mathrm{p}<0.01$; Fig. $2 \mathrm{C}$ ).

We next quantified pericyte cell coverage of the cerebrovascular wall, which is the percentage of the vascular wall covered by pericyte cell processes. Quantitative immunofluorescent analysis demonstrated a statistically significant $52.3 \%$ reduction in the vascular surface area covered by CD13-positive pericyte cell processes (bAVMs $39.4 \% \pm 2.8 \%$; NVLCs $82.7 \% \pm 2.1 \%$; p < 0.01; Fig. 2D). These analyses were confirmed utilizing the independent pericyte cell marker PDGFR $\beta$, which demonstrated a similar statistically significant $49.9 \%$ reduction in PDGFR $\beta$ positive pericyte cell coverage between bAVMs and NVLCs (bAVMs 40.6\% + 2.5\%; NVLCs $81.3 \% \pm 2.3 \%$; p < 0.01 ; Fig. 2E). These data suggest that both pericyte cell number and coverage of the vascular wall are reduced in sporadic human bAVMs.

\section{Magnitude of Pericyte Reductions in Ruptured bAVMs}

Having demonstrated a reduction in pericyte number

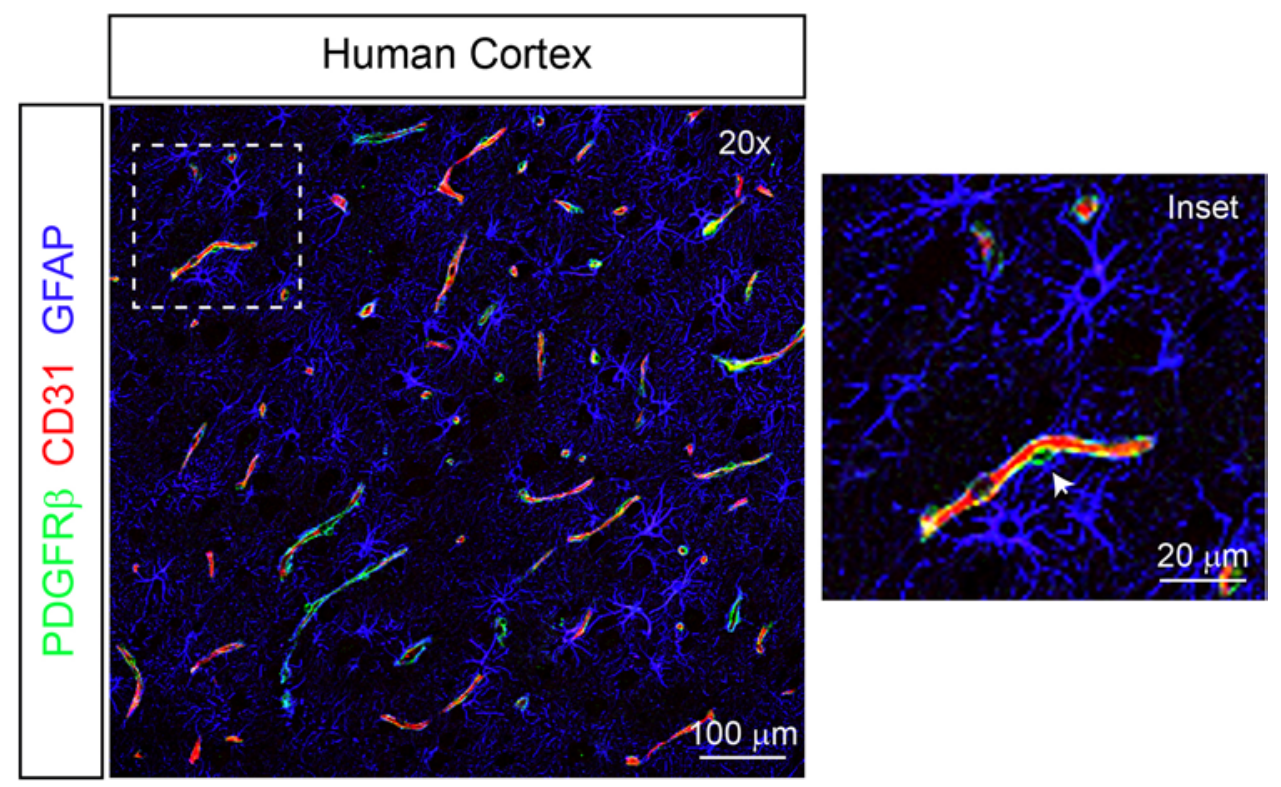

FIG. 1. Cytoarchitecture of the cerebrovasculature in the human cortex. Representative low-magnification confocal microscopy image depicting the close structural relationship among pericytes (PDGFR $\beta$, green), endothelial cells (CD31, red), and astrocytes (GFAP, blue) in human cortex. Inset features a higher-magnification view demonstrating a pericyte cell body (arrowhead) and contiguous stellate process, which ensheathe the endothelial wall. 

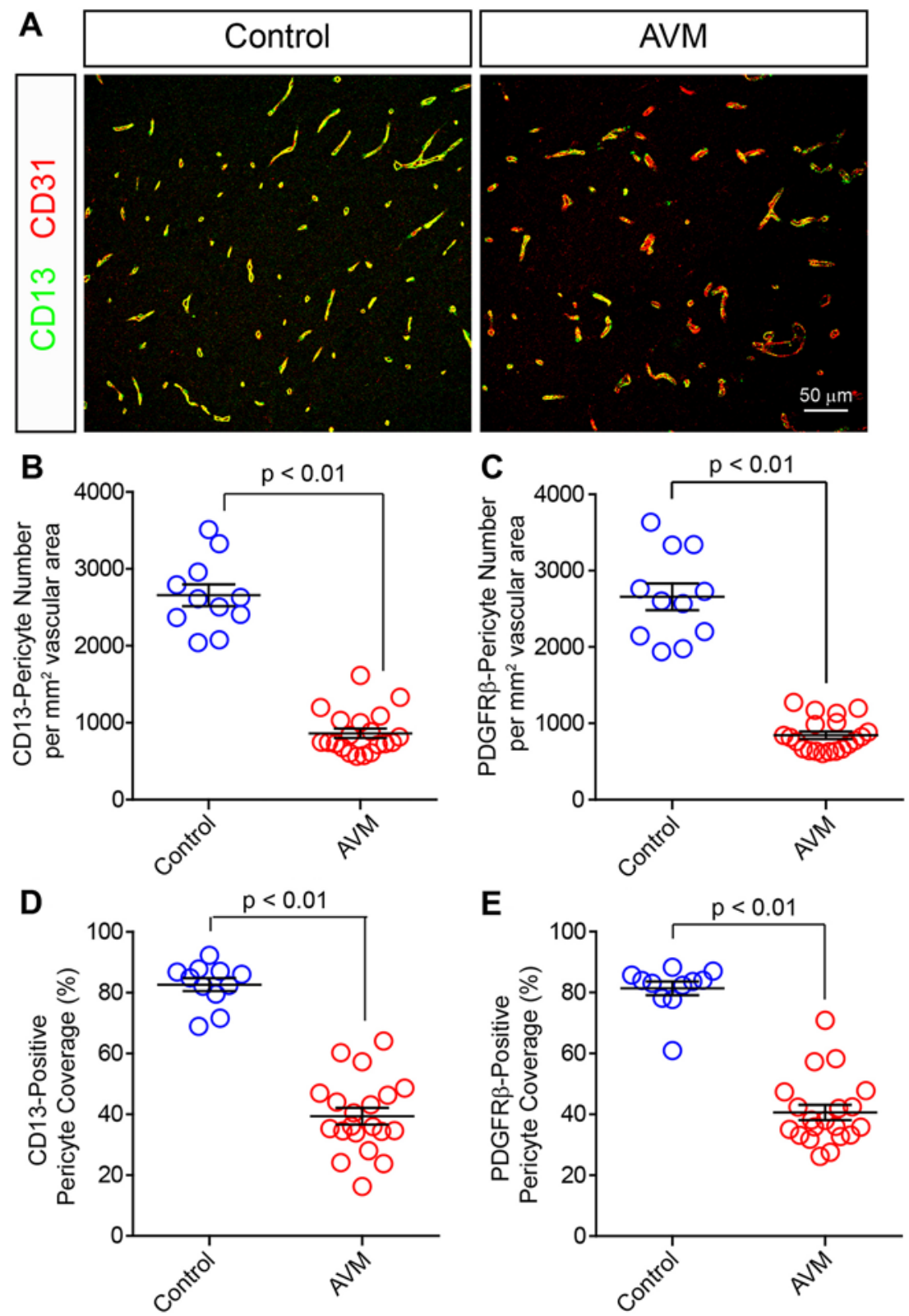

FIG. 2. Vascular pericytes are reduced in human bAVMs. Representative confocal microscopy analysis (A) of CD13-positive pericytes (green) and CD31-positive endothelium (red) in human AVMs and temporal cortex from NVLCs. Graphs showing quantification of CD13-positive (B) and PDGFRß-positive (C) pericyte cells per square millimeter vascular surface area in NVLCs and bAVMs. Graphs showing quantification of pericyte coverage of the vascular wall utilizing CD13 (D) and PDGFR (E) immunolabeling of pericyte cell processes. Values in the graphs are expressed as the mean \pm standard error of the mean.

and coverage in bAVMs, we used similar analyses to determine whether differences in pericyte populations existed between ruptured and unruptured bAVMs. No statistically significant difference in the CD13- and PDGFR $\beta$-positive pericyte cell number was demonstrated between unruptured and ruptured bAVM cohorts (Table 2). However, quantitative immunofluorescent analysis of the vascular coverage of CD13- and PDGFR $\beta$-positive cell processes showed $25.7 \%$ and $20.7 \%$ greater reductions in pericyte coverage, respectively, in ruptured than in unruptured bAVMs. These data suggest that pericyte coverage, but not pericyte number, is further reduced in bAVMs with clinically significant rupture.

\section{Reductions in Pericyte Populations in Unruptured bAVMs}

Silent microhemorrhage has been proposed as a risk factor for future bAVM rupture and may serve as a surrogate for the relative instability of a subset of bAVMs.,12 Therefore, we investigated whether the observed reductions in pericytes correlated with the extent of acute and chronic microhemorrhage in the unruptured bAVM group. Quantitative immunofluorescent analysis demonstrated a 
TABLE 2. Pericyte abundance and coverage values in NVLCs and bAVMs

\begin{tabular}{|c|c|c|c|c|c|}
\hline \multirow[b]{2}{*}{ Parameter } & \multirow[b]{2}{*}{ NVLCs } & \multicolumn{3}{|c|}{ AVMs } & \multirow{2}{*}{$\begin{array}{c}p \\
\text { Value* }\end{array}$} \\
\hline & & Total & Unruptured & Ruptured & \\
\hline \multicolumn{6}{|l|}{ Pericyte no. } \\
\hline PDGFR $\beta$-positive pericytes (per mm² endothelium) & $2658.1 \pm 174.8$ & $845.6 \pm 47.9 \dagger$ & $844.9 \pm 63.8$ & $842.8 \pm 77.4$ & 0.99 \\
\hline CD13-positive pericytes (per mm² endothelium) & $2657.0 \pm 140.7$ & $862.2 \pm 61.4 \dagger$ & $832.3 \pm 71.7$ & $907.1 \pm 114.1$ & 0.59 \\
\hline \multicolumn{6}{|l|}{ Pericyte coverage } \\
\hline \% PDGFR $\beta$-positive pericyte coverage & $81.3 \pm 2.3$ & $40.6 \pm 2.5 \dagger$ & $44.3 \pm 3.6$ & $35.1 \pm 2.0$ & 0.04 \\
\hline$\%$ CD13-positive pericyte coverage & $82.7 \pm 2.1$ & $39.4 \pm 2.8 \dagger$ & $43.9 \pm 3.2$ & $32.6 \pm 3.4$ & 0.03 \\
\hline
\end{tabular}

statistically significant 15.5 -fold increase in extravascular hemoglobin consistent with acute microhemorrhage in unruptured bAVMs compared with that in NVLCs (mean extravascular hemoglobin integrated density: bAVMs 1,206,583 \pm 307,333 units; NVLCs 77,742.4 \pm 30,829 units; $\mathrm{p}<0.01$; Fig. 3A and B). Within the unruptured bAVMs, pericyte coverage correlated negatively with the extent of microhemorrhage (CD13-positive pericyte coverage: $r=$ $-0.93, \mathrm{p}<0.01$; PDGFR $\beta$-positive pericyte coverage: $\mathrm{r}=$ $-0.87, \mathrm{p}<0.01$; Fig. $3 \mathrm{C}$ and D). This suggests that lower pericyte coverage is associated with greater microhemorrhage within unruptured bAVMs.

To confirm these results, we investigated whether a similar relationship was present between pericyte coverage and chronic microhemorrhage as evidence by Prussian blue-positive hemosiderin deposits. This analysis demonstrated a statistically significant 21.5-fold increase in perivascular hemosiderin deposits in unruptured bAVMs compared with those in NVLCs (bAVMs $0.46 \pm 0.11$ deposits; NVLCs $0.02 \pm 0.01$ deposits; $\mathrm{p}<0.01$; Fig. 4A and B).
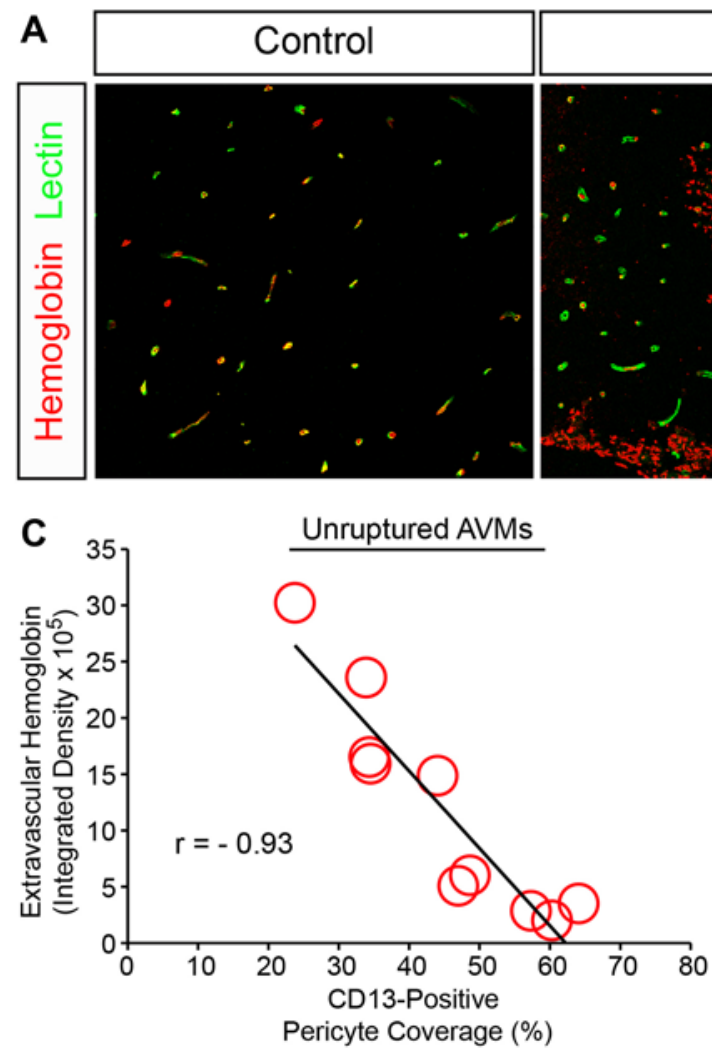
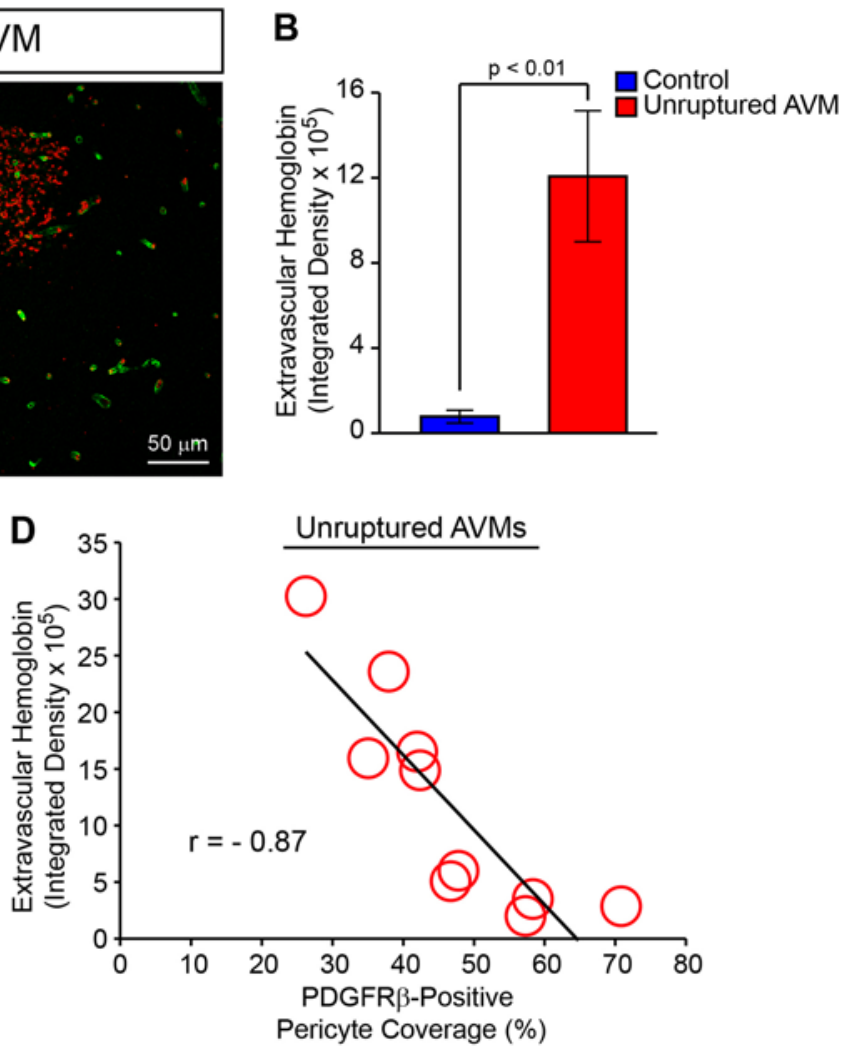

FIG. 3. Reductions in vascular pericytes are associated with acute cerebral microhemorrhage in unruptured bAVMs. A: Representative confocal microscopy analysis of hemoglobin (red) and lectin-positive endothelium in temporal cortex from NVLCs and bAVMs. Yellow indicates colocalized intravascular hemoglobin. B: Graph demonstrating quantification of extravascular hemoglobin immunofluorescent signal from NVLCs (b/ue) and unruptured bAVMs (red). Values are expressed as the mean \pm standard error of the mean. C and D: Graphic representation of correlation between CD13- or PDGFRß-positive pericyte coverage and extravascular hemoglobin in unruptured bAVM tissue specimens. 

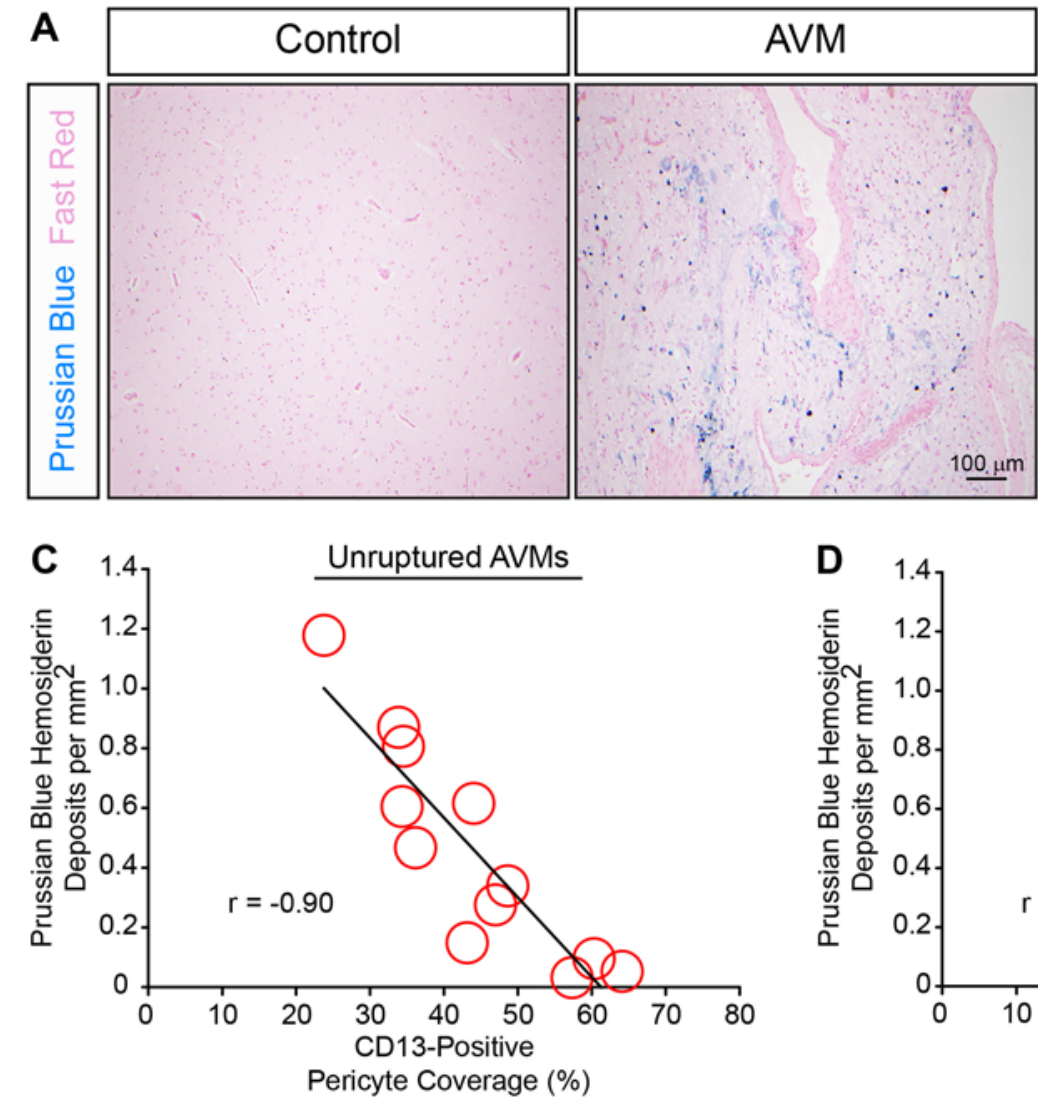
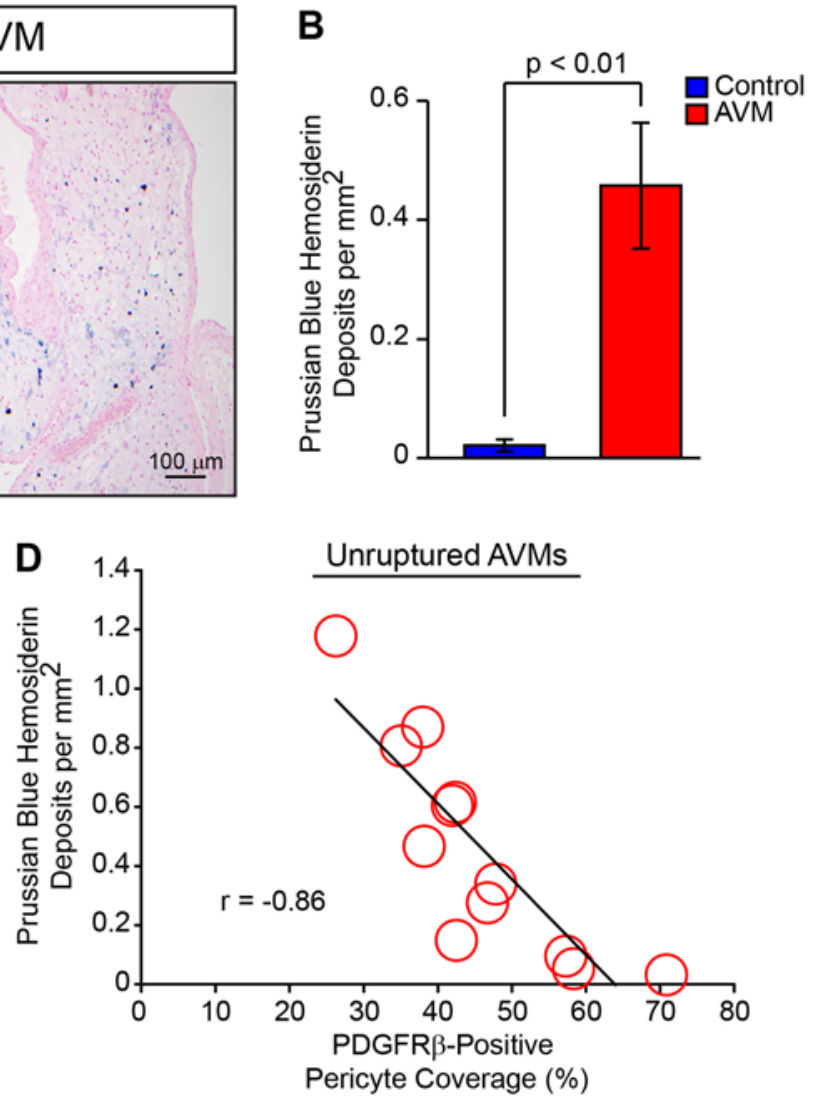

FIG. 4. Pericyte reductions are associated and negatively correlated with chronic microhemorrhage in unruptured bAVMs. A: Representative bright-field microscopy analysis of Prussian blue-positive hemosiderin deposits (b/ue) with Nuclear fast red counterstain (pink) in bAVMs and temporal cortex from NVLCs. B: Graph demonstrating quantification of Prussian blue-positive hemosiderin deposits from NVLCs (b/ue) and unruptured bAVMs (red). Values are expressed as the mean \pm standard error of the mean. C and D: Graphic representations of correlation between CD13- or PDGFR $\beta$-positive pericyte coverage and hemosiderin deposition in unruptured bAVM tissue specimens.

As observed with acute microhemorrhage, pericyte coverage correlated negatively with Prussian blue-positive hemosiderin deposits in unruptured bAVMs (CD13-positive pericyte coverage: $\mathrm{r}=-0.90, \mathrm{p}<0.01$; PDGFR $\beta$-positive pericyte coverage: $r=-0.86, p<0.01$; Fig. $4 \mathrm{C}$ and D). Collectively, these data suggest that lower pericyte coverage is associated with greater microhemorrhage in unruptured bAVMs.

\section{bAVM Pericyte Reductions and Nidal Blood Flow}

In addition to stabilizing the cerebrovasculature, pericytes contribute to the regulation of regional cerebral blood flow. $6,13,17,22$ Therefore, we used syngo iFlow technology on preintervention angiograms when available to quantify blood flow through the bAVM nidus to determine whether reductions in bAVM pericyte populations are associated with alterations in nidal blood flow. Within bAVMs with compatible preoperative angiograms (12 cases), pericyte coverage correlated positively with the MTT of injected contrast through the nidus (CD13-positive pericyte coverage: $r=0.60, p<0.05$; PDGFR $\beta$-positive pericyte coverage: $r=0.63, p<0.05$; Fig. 5). This suggests that greater pericyte coverage is associated with a longer transit time or slow rates of blood flow through the bAVM nidus. Conversely, bAVMs with the greatest loss of pericytes demonstrated shorter transit times or faster rates of nidal blood flow. No statistically significant correlations were observed with time to peak flow within bAVM feeding arteries or draining veins (data not shown).

\section{Discussion}

In the present report, we demonstrate that a reduction in pericytes is associated with both vascular instability and altered hemodynamics in human bAVMs. Prior qualitative works have suggested that mural cells may be reduced in human bAVMs and/or rodent models of bAVM ${ }^{8,34}$ However, using 2 independent and established pericyte markers-CD13 and PDGFR $\beta$-we provide the first quantitative evidence of a significant reduction in both pericyte cell number and coverage in human sporadic bAVMs. Consistent with prior reports on other pathologies, the magnitude of deficiency in the pericyte cell number exceeds what is observed with cell coverage. ${ }^{6,30,40}$ Numerous reports have demonstrated that reductions in pericyte cell coverage are of greatest biological consequence and best correlate with indices of pericyte cell function. ${ }^{3,6,9}$ This suggests in part that the remaining pericytes may hyper- 

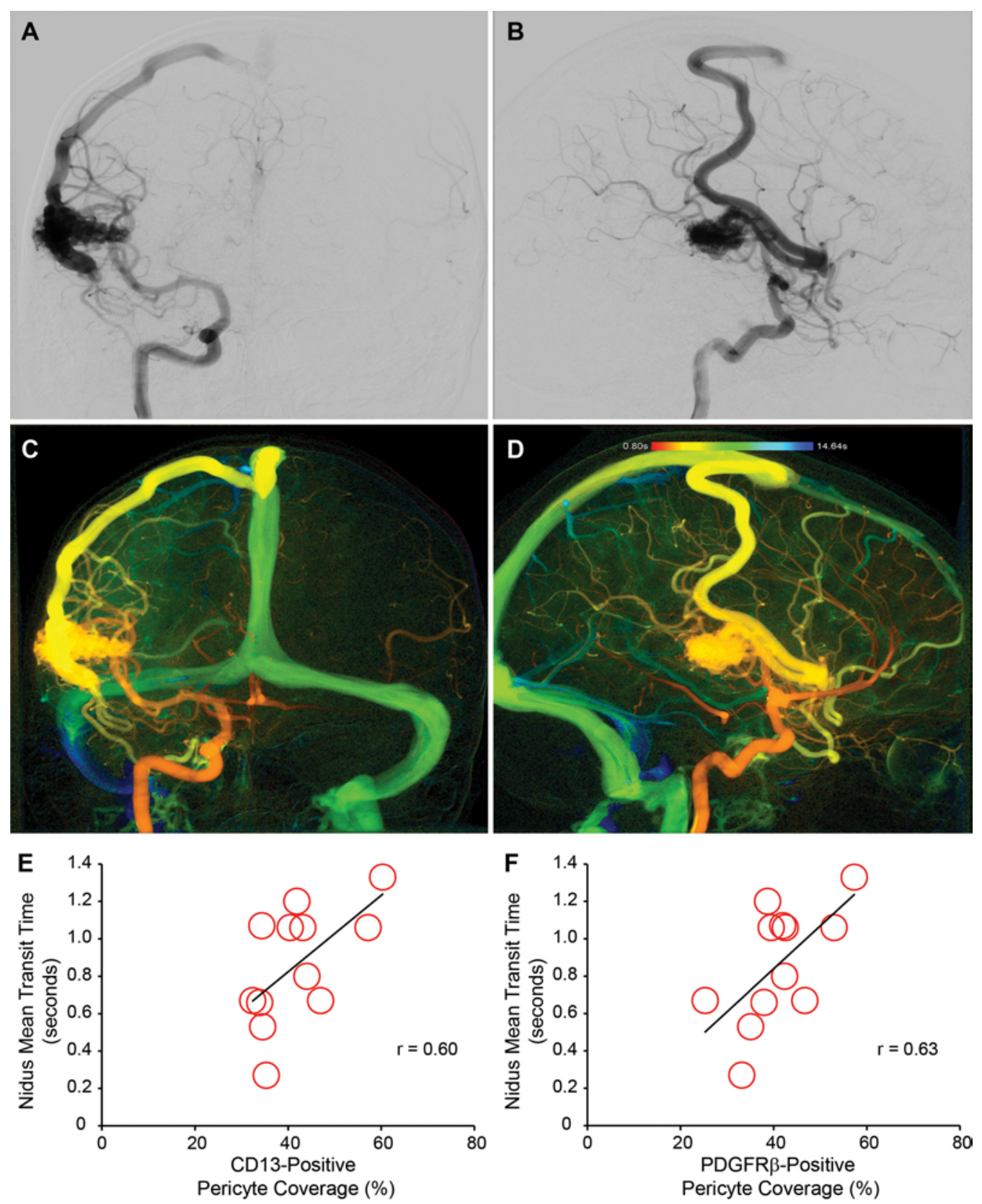

FIG. 5. Pericyte reductions positively correlate with blood flow through the bAVM nidus. Representative preoperative angiograms, anteroposterior $(\mathbf{A})$ and lateral $(\mathbf{B})$ views of left internal carotid artery injection, demonstrating Spetzler-Martin grade II, supplementary grade 4 right lateral temporal bAVM. Representative syngo iFlow postprocessing of preintervention angiogram to determine mean iFlow transit time of bAVM nidus, anteroposterior (C) and lateral (D) views of left internal carotid artery injection. Graphic representations of correlation between CD13-positive (E) or PDGFRß-positive (F) pericyte coverage and MTT of blood flow through the bAVM nidus using iFlow analysis. Each point depicts an individual subject.

trophy and/or branch additional processes as a mechanism of potential compensation.

Pericytes exert a multitude of stabilizing effects on the cerebrovascular wall in vivo, including induction and maintenance of endothelial tight junctional protein complexes, deposition of perivascular extracellular matrix proteins, and inhibition of degradative enzymes, for example, matrix metalloproteinases. ${ }^{3,67,7,35,38}$ Genetic depletion of brain pericytes in rodents results in cerebral vessels that display focal and/or diffuse dilations with increased tor- tuosity, which are prone to rupture and chronically leak circulating blood-derived plasma proteins. ${ }^{3,6,10,16,20,21,35,36}$ In rodent bAVM models with deficient transforming growth factor- $\beta$ (TGF- $\beta$ ) signaling through genetic depletion of vascular activin receptor-like kinase 1 (Alkl), a reduction in brain pericytes was correlated with leakage of circulating blood-derived fibrin, suggesting a compromised BBB. ${ }^{8}$ However, the consequences of pericyte deficiency in human bAVMs are presently unknown.

To begin to elucidate the role of pericyte deficiency in 
human bAVMs, we adopted a correlative approach to define hypothesis-driven associations with known pericyte functions. Consistent with a potential stabilizing role of pericytes, reductions in pericyte coverage were greatest in bAVMs with prior rupture resulting in intracerebral hemorrhage. In unruptured bAVMs, clinically silent microhemorrhage portends a higher risk of future rupture and may represent a transitional state in the continuum of destabi-

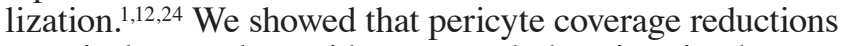
negatively correlate with acute and chronic microhemorrhages in unruptured human bAVMs. Although associative in nature, these data suggest that a greater loss of pericytes may contribute to a greater microhemorrhage burden and/ or likelihood of rupture. However, the threshold of pericyte loss required to evoke vascular destabilization in bAVMs and/or other disease entities has yet to be elucidated.

In addition to stabilizing the vasculature, pericytes help to regulate resting cerebral blood flow and blood flow responses to neuronal activation - a concept known as "neurovascular coupling."',13,17,22 Pericytes express contractile proteins and have been shown to modulate vascular diameter both at rest and in response to a myriad of molecular cues. . $13,25,41,42$ In rodent models of bAVM, in vivo timelapse multiphoton microscopy has demonstrated that progressive dilation of capillary beds contributes to arteriovenous shunting. ${ }^{23}$ When quantitative iFlow analysis was applied to preintervention angiograms, we found that pericyte coverage positively correlates with the MTT of blood flow through the nidus in human bAVMs. This means that a greater reduction in pericyte coverage is associated with a reduced MTT, or a faster rate of flow, through the bAVM nidus. Pericyte deficiency has been associated with increases in vascular diameter and/or loss of vasomotor tone in vivo. ${ }^{3,16,17}$ Whether this finding in human bAVMs is the result of changes in vascular diameter and/or vascular tone remains to be determined. Similarly, the ramifications of these hemodynamic changes on lesion stability and/or remodeling require future study.

It should be noted that pericyte deficiency is not unique to human bAVMs and has been previously reported in a number of human neurological diseases with vascular pathology, including Alzheimer's disease, ${ }^{14,27,30}$ amyotrophic lateral sclerosis, ${ }^{39,40}$ cavernous malformations, ${ }^{29,33}$ and prenatal intraventricular hemorrhage. ${ }^{5,20}$ Pericyte deficiency may therefore represent a common downstream pathway of vascular destabilization in the central nervous system. During brain angiogenesis, the recruitment of pericytes to nascent endothelial cell tubes relies on a complex interplay of multiple signal transduction cascades, including platelet-derived growth factor B (PDGF-B), TGF- $\beta$, Notch, vascular endothelial growth factor, and angiopoietin cell sig-

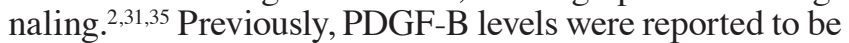
undetectable in a majority of bAVMs. ${ }^{15}$ However, the inciting molecular event(s) for pericyte reductions in human bAVMs and whether it is unique among disease states with pericyte deficiency remain to be determined. In other disease states, such as hereditary hemorrhagic telangiectasia, pericytes can be therapeutically manipulated to stabilize the vascular wall and reduce bleeding. ${ }^{19,32}$ Future studies are needed to evaluate the therapeutic potential of manipulating pericytes or, more broadly, mural cells in bAVMs.
This study was not without limitations, and the results should be appropriately framed. Experiments were conducted on resected bAVMs and may therefore reflect a selection bias toward more aggressive lesions that were clinically detected. Comparisons were also made utilizing an NVLC of resected temporal lobe from patients with medically refractory temporal lobe epilepsy and not from brain completely devoid of pathology. Prior reports have demonstrated that pericyte populations do not vary significantly within brain regions, and reported values for controls are in good agreement with previously reported values from postmortem nonepileptic human brain samples from multiple brain regions..$^{30,37,38}$ Nonetheless, the contribution of regional variability in pericyte abundance to the observed findings cannot be completely excluded. Finally, all relationships are associative and correlative in nature and do not imply causality. Future studies in rodent models are needed to more clearly delineate a causal relationship between pericyte loss and vascular changes in bAVMs.

\section{Conclusions}

Brain pericyte number and coverage are reduced in sporadic human bAVMs. Pericyte reductions are greatest in bAVMs with clinically significant hemorrhage and are associated with a greater microhemorrhage burden in unruptured cases. Pericyte reduction also correlates with faster blood flow through the bAVM nidus. This suggests that pericytes are associated with and may contribute to vascular fragility and hemodynamic changes in human bAVMs. Future studies with animal models are needed to better characterize the role of pericytes in AVM pathogenesis and to determine whether these cells can be therapeutically targeted to promote bAVM stabilization.

\section{Acknowledgments}

This work was supported by the following grants: NIH grant no. RO1 NS034949 (H.K.); NIH grant nos. R01-NS027713 and R01HL122774 and Michael Ryan Zodda Foundation (H.S.); National Natural Science Foundation of China 81500995 (X.C.); and Congress of Neurological Surgeons Christopher C. Getch Fellowship (B.P.W.).

\section{References}

1. Abla AA, Nelson J, Kim H, Hess CP, Tihan T, Lawton MT: Silent arteriovenous malformation hemorrhage and the recognition of "unruptured" arteriovenous malformation patients who benefit from surgical intervention. Neurosurgery 76:592-600, 2015

2. Armulik A, Genové G, Betsholtz C: Pericytes: developmental, physiological, and pathological perspectives, problems, and promises. Dev Cell 21:193-215, 2011

3. Armulik A, Genové G, Mäe M, Nisancioglu MH, Wallgard E, Niaudet C, et al: Pericytes regulate the blood-brain barrier. Nature 468:557-561, 2010

4. Attia W, Tada T, Hongo K, Nagashima H, Takemae T, Tanaka $\mathrm{Y}$, et al: Microvascular pathological features of immediate perinidal parenchyma in cerebral arteriovenous malformations: giant bed capillaries. J Neurosurg 98:823-827, 2003

5. Ballabh P, Braun A, Nedergaard M: The blood-brain barrier: an overview: structure, regulation, and clinical implications. Neurobiol Dis 16:1-13, 2004

6. Bell RD, Winkler EA, Sagare AP, Singh I, LaRue B, Deane 
$\mathrm{R}$, et al: Pericytes control key neurovascular functions and neuronal phenotype in the adult brain and during brain aging. Neuron 68:409-427, 2010

7. Bell RD, Winkler EA, Singh I, Sagare AP, Deane R, Wu Z, et al: Apolipoprotein E controls cerebrovascular integrity via cyclophilin A. Nature 485:512-516, 2012

8. Chen W, Guo Y, Walker EJ, Shen F, Jun K, Oh SP, et al: Reduced mural cell coverage and impaired vessel integrity after angiogenic stimulation in the Alk1-deficient brain. Arterioscler Thromb Vasc Biol 33:305-310, 2013

9. Daneman R, Zhou L, Kebede AA, Barres BA: Pericytes are required for blood-brain barrier integrity during embryogenesis. Nature 468:562-566, 2010

10. Enge M, Bjarnegård M, Gerhardt H, Gustafsson E, Kalén M, Asker N, et al: Endothelium-specific platelet-derived growth factor-B ablation mimics diabetic retinopathy. EMBO J 21:4307-4316, 2002

11. Gross BA, Du R: Natural history of cerebral arteriovenous malformations: a meta-analysis. J Neurosurg 118:437-443, 2013

12. Guo Y, Saunders T, Su H, Kim H, Akkoc D, Saloner DA, et al: Silent intralesional microhemorrhage as a risk factor for brain arteriovenous malformation rupture. Stroke 43:12401246,2012

13. Hall CN, Reynell C, Gesslein B, Hamilton NB, Mishra A, Sutherland BA, et al: Capillary pericytes regulate cerebral blood flow in health and disease. Nature 508:55-60, 2014

14. Halliday MR, Rege SV, Ma Q, Zhao Z, Miller CA, Winkler EA, et al: Accelerated pericyte degeneration and bloodbrain barrier breakdown in apolipoprotein E4 carriers with Alzheimer's disease. J Cereb Blood Flow Metab 36:216227, 2016

15. Hashimoto T, Wu Y, Lawton MT, Yang GY, Barbaro NM, Young WL: Coexpression of angiogenic factors in brain arteriovenous malformations. Neurosurgery 56:1058-1065, 2005

16. Hellström M, Gerhardt H, Kalén M, Li X, Eriksson U, Wolburg H, et al: Lack of pericytes leads to endothelial hyperplasia and abnormal vascular morphogenesis. J Cell Biol 153:543-553, 2001

17. Kisler K, Nelson AR, Rege SV, Ramanathan A, Wang Y, Ahuja A, et al: Pericyte degeneration leads to neurovascular uncoupling and limits oxygen supply to brain. Nat Neurosci 20:406-416, 2017

18. Lawton MT, Rutledge WC, Kim H, Stapf C, Whitehead KJ, Li DY, et al: Brain arteriovenous malformations. Nat Rev Dis Primers 1:15008, 2015

19. Lebrin F, Srun S, Raymond K, Martin S, van den Brink S, Freitas C, et al: Thalidomide stimulates vessel maturation and reduces epistaxis in individuals with hereditary hemorrhagic telangiectasia. Nat Med 16:420-428, 2010

20. Li F, Lan Y, Wang Y, Wang J, Yang G, Meng F, et al: Endothelial Smad4 maintains cerebrovascular integrity by activating N-cadherin through cooperation with Notch. Dev Cell 20:291-302, 2011

21. Lindahl P, Johansson BR, Levéen P, Betsholtz C: Pericyte loss and microaneurysm formation in PDGF-B-deficient mice. Science 277:242-245, 1997

22. Mishra A, Reynolds JP, Chen Y, Gourine AV, Rusakov DA, Attwell D: Astrocytes mediate neurovascular signaling to capillary pericytes but not to arterioles. Nat Neurosci 19:1619-1627, 2016

23. Murphy PA, Kim TN, Huang L, Nielsen CM, Lawton MT, Adams RH, et al: Constitutively active Notch4 receptor elicits brain arteriovenous malformations through enlargement of capillary-like vessels. Proc Natl Acad Sci U S A 111:18007-18012, 2014

24. Pekmezci M, Nelson J, Su H, Hess C, Lawton MT, Sonmez $\mathrm{M}$, et al: Morphometric characterization of brain arteriovenous malformations for clinical and radiological studies to identify silent intralesional microhemorrhages. Clin Neuropathol 35:114-121, 2016

25. Peppiatt CM, Howarth C, Mobbs P, Attwell D: Bidirectional control of CNS capillary diameter by pericytes. Nature 443:700-704, 2006

26. Rangel-Castilla L, Russin JJ, Martinez-Del-Campo E, Soriano-Baron H, Spetzler RF, Nakaji P: Molecular and cellular biology of cerebral arteriovenous malformations: a review of current concepts and future trends in treatment. Neurosurg Focus 37(3):E1, 2014

27. Sagare AP, Bell RD, Zhao Z, Ma Q, Winkler EA, Ramanathan A, et al: Pericyte loss influences Alzheimer-like neurodegeneration in mice. Nat Commun 4:2932, 2013

28. Sato S, Kodama N, Sasaki T, Matsumoto M, Ishikawa T: Perinidal dilated capillary networks in cerebral arteriovenous malformations. Neurosurgery 54:163-170, 2004

29. Schulz GB, Wieland E, Wüstehube-Lausch J, Boulday G, Moll I, Tournier-Lasserve E, et al: Cerebral cavernous malformation-1 protein controls DLL4-Notch3 signaling between the endothelium and pericytes. Stroke 46:1337-1343, 2015

30. Sengillo JD, Winkler EA, Walker CT, Sullivan JS, Johnson M, Zlokovic BV: Deficiency in mural vascular cells coincides with blood-brain barrier disruption in Alzheimer's disease. Brain Pathol 23:303-310, 2013

31. Sweeney MD, Ayyadurai S, Zlokovic BV: Pericytes of the neurovascular unit: key functions and signaling pathways. Nat Neurosci 19:771-783, 2016

32. Thalgott J, Dos-Santos-Luis D, Lebrin F: Pericytes as targets in hereditary hemorrhagic telangiectasia. Front Genet 6:37, 2015

33. Tu J, Stoodley MA, Morgan MK, Storer KP: Ultrastructural characteristics of hemorrhagic, nonhemorrhagic, and recurrent cavernous malformations. J Neurosurg 103:903-909, 2005

34. Tu J, Stoodley MA, Morgan MK, Storer KP: Ultrastructure of perinidal capillaries in cerebral arteriovenous malformations. Neurosurgery 58:961-970, 2006

35. Winkler EA, Bell RD, Zlokovic BV: Central nervous system pericytes in health and disease. Nat Neurosci 14:1398-1405, 2011

36. Winkler EA, Bell RD, Zlokovic BV: Lack of Smad or Notch leads to a fatal game of brain pericyte hopscotch. Dev Cell 20:279-280, 2011

37. Winkler EA, Bell RD, Zlokovic BV: Pericyte-specific expression of PDGF beta receptor in mouse models with normal and deficient PDGF beta receptor signaling. Mol Neurodegener 5:32, 2010

38. Winkler EA, Sengillo JD, Bell RD, Wang J, Zlokovic BV: Blood-spinal cord barrier pericyte reductions contribute to increased capillary permeability. J Cereb Blood Flow Metab 32:1841-1852, 2012

39. Winkler EA, Sengillo JD, Sagare AP, Zhao Z, Ma Q, Zuniga E, et al: Blood-spinal cord barrier disruption contributes to early motor-neuron degeneration in ALS-model mice. Proc Natl Acad Sci U S A 111:E1035-E1042, 2014

40. Winkler EA, Sengillo JD, Sullivan JS, Henkel JS, Appel SH, Zlokovic BV: Blood-spinal cord barrier breakdown and pericyte reductions in amyotrophic lateral sclerosis. Acta Neuropathol 125:111-120, 2013

41. Yemisci M, Gursoy-Ozdemir Y, Vural A, Can A, Topalkara $\mathrm{K}$, Dalkara T: Pericyte contraction induced by oxidativenitrative stress impairs capillary reflow despite successful opening of an occluded cerebral artery. Nat Med 15:10311037, 2009

42. Zeisel A, Muñoz-Manchado AB, Codeluppi S, Lönnerberg $\mathrm{P}$, La Manno G, Juréus A, et al: Brain structure. Cell types in the mouse cortex and hippocampus revealed by single-cell RNA-seq. Science 347:1138-1142, 2015

43. Zhang R, Zhu W, Su H: Vascular integrity in the pathogen- 
esis of brain arteriovenous malformation. Acta Neurochir Suppl 121:29-35, 2016

44. Zhao Z, Nelson AR, Betsholtz C, Zlokovic BV: Establishment and dysfunction of the blood-brain barrier. Cell 163:1064-1078, 2015

\section{Disclosures}

The authors report no conflict of interest concerning the materials or methods used in this study or the findings specified in this paper.

\section{Author Contributions}

Conception and design: Lawton, Winkler, Tihan, Su, Kim, Wal- cott. Acquisition of data: Winkler, Birk, Burkhardt, Chen, Guo, Rutledge, Lasker, Partow, Tihan, Chang. Analysis and interpretation of data: Winkler, Birk, Burkhardt, Su, Kim, Walcott. Drafting the article: Winkler, Burkhardt, Chen, Yue, Lasker. Critically revising the article: Lawton, Winkler, Rutledge, Su, Kim, Walcott Reviewed submitted version of manuscript: Lawton, Winkler, Su, Kim, Walcott. Approved the final version of the manuscript on behalf of all authors: Lawton. Statistical analysis: Winkler, Yue. Study supervision: Lawton.

\section{Correspondence}

Michael T. Lawton: Barrow Neurological Institute, Phoenix, AZ. michael.lawton@barrowbrainandspine.com. 